\title{
Antibiogram profiles and efficacy of antibiotic regimens of bacterial isolates from chronic osteomyelitis of the appendicular skeleton: A developing-world perspective
}

\author{
N Ferreira, ${ }^{1}$ BSc, MB ChB, FC Orth (SA), MMed (Orth), PhD; K Reddy, ${ }^{2}$ MB ChB, FC Path (SA) Micro, MMed (Microb Path), \\ MSc (Clin Epi), Dip MH, Dip Obst, Dip HIV Man (SA); R G Venter, ${ }^{1}$ MB ChB, FC Orth (SA), MMed (Orth); C M Centner, ${ }^{3}$ MB ChB, MSc, \\ MMed, FC Path (SA) Micro; M Laubscher, ${ }^{4}$ MB ChB, Dip PEC, FC Orth (SA), MMed (Orth)
${ }^{1}$ Division of Orthopaedic Surgery, Department of Surgical Sciences, Tygerberg Hospital and Faculty of Medicine and Health Sciences, Stellenbosch University, Cape Town, South Africa
${ }^{2}$ Division of Medical Microbiology and Immunology and National Health Laboratory Service Tygerberg, Faculty of Medicine and Health Sciences, Stellenbosch University, Cape Town, South Africa
${ }^{3}$ Division of Medical Microbiology, Faculty of Health Sciences, University of Cape Town; and National Health Laboratory Service, Groote Schuur Hospital, South Africa
${ }^{4}$ Orthopaedic Research Unit, Division of Orthopaedic Surgery, Department of Surgery, Faculty of Health Sciences, University of Cape Town, South Africa

Corresponding author: N Ferreira (nferreira@sun.ac.za)

\begin{abstract}
Background. Empirical antibiotic strategies in the treatment of chronic osteomyelitis should ideally be based on local microbiological antibiograms.

Objectives. To review the antibiogram profiles of bacterial isolates of patients undergoing surgical treatment for chronic osteomyelitis and identify the most appropriate empirical antibiotic strategy.

Methods. A retrospective review of clinical records and microbial culture reports was performed for all patients who underwent treatment for chronic osteomyelitis at two orthopaedic units in Western Cape Province, South Africa, between March 2016 and December 2019. Reported antibiotic susceptibility data were used to predict the potential efficacy of different empirical antibiotic regimens, according to underlying aetiology (fracture related, contiguous, haematogenous).

Results. Two hundred patients with chronic osteomyelitis of the appendicular skeleton underwent surgical management. Antibiogram profiles for 218 organisms, isolated from 169 patients, were evaluated. Staphylococcus aureus (41\%) and Enterobacterales (30\%) were the most common organisms isolated. The combinations of meropenem plus vancomycin, and piperacillin-tazobactam plus amikacin plus vancomycin, as empirical postoperative antibiotics would both effectively treat $78 \%$ of chronic osteomyelitis cases overall. The most effective practical oral combinations were co-amoxiclav plus ciprofloxacin (61\%) and co-trimoxazole plus ciprofloxacin (61\%).

Conclusions. This study reports antibiogram profiles in the developing-world setting that could potentially guide empirical antibiotic choices in the management of chronic osteomyelitis.
\end{abstract}

S Afr Med J 2021;111(7):642-648. https://doi.org/10.7196/SAMJ.2021.v111i7.15516

Chronic osteomyelitis is notoriously difficult to eradicate, and high treatment failure rates have been reported in the literature. ${ }^{[1,2]}$ Although no evidence-based treatment guidelines exist for the management of chronic osteomyelitis, the ideal treatment strategy can be outlined as judicious resection of all necrotic tissue, dead-space management, and neovascularisation of the debridement site followed by soft-tissue and bony reconstruction as required. ${ }^{[3-7]}$ Antibiotic therapy is empirically initiated as an adjunct to surgical management, and then continued as prolonged culture-specific (targeted) therapy. ${ }^{[3]}$

In the absence of non-invasive sampling methods to ascertain the microbiological profile of osteomyelitis, the choice of empirical antibiotic therapy to initiate is often aimed at the most probable infecting organism, in conjunction with current international reports. ${ }^{[8]}$ As the organism and antibiotic susceptibility profiles conceivably differ between geographical regions, empirical antibiotic strategies should ideally be based on local microbiological antibiograms. There are limited data available on the local pathogen profiles and antibiograms in developing countries, including South Africa (SA) ${ }^{[9]}$

\section{Objectives}

To review the antibiogram profiles of bacterial isolates from patients with chronic osteomyelitis of the appendicular skeleton who underwent surgical treatment at two high-volume dedicated musculoskeletal infection units in Western Cape Province, South Africa (SA). Secondary objectives were to evaluate potential empirical antibiotic regimens according to the cause of the osteomyelitis, i.e. fracture-related infection, haematogenous or contiguous spread.

\section{Methods}

A retrospective review of clinical records, microbial culture reports and antibiotic susceptibility data was performed for all patients of any age who underwent treatment for chronic osteomyelitis between 1 March 2016 and 31 December 2019. Acute infections (septic arthritis and early deep surgical infections), spinal infections and periprosthetic joint infections were excluded.

At each institution, all patients were operated on by one of two musculoskeletal infection specialist surgeons (NF or ML) during the study period. Where cultures were taken from multiple surgical 
interventions, only results from the definitive surgical procedure were included. Ethical and hospital board approval (ref. no. N18/08/082) were obtained prior to data collection. Chronic osteomyelitis was defined as at least 6 months of symptoms, as well as one of the following:

- sinus, abscess or purulence at the time of surgery

- histological features suggestive of chronic osteomyelitis.

Patients were stratified according to the original pathology into fracturerelated infection, contiguous infection and haematogenous infection subgroups. Fracture-related infection was defined according to the criteria proposed by Metsemakers et al. ${ }^{[10]}$ and consisted of one of the following:

- fistula, sinus or wound breakdown (that communicates to the bone or implant)

- purulent drainage or the presence of pus during surgery

- presence of micro-organisms in deep-tissue specimens confirmed by histopathological examination.

Contiguous osteomyelitis was defined as an osseous infection that resulted from extension from an adjacent soft-tissue infection. Haematogenous infection was defined as chronic osteomyelitis that resulted from a de novo infection without any local predisposing factors such as trauma, surgery or neighbouring infection.

In all cases, intraoperatively collected deep samples of infected tissue and/or biofilm were submitted for bacterial culture. Samples were submitted to the relevant on-site National Health Laboratory Service diagnostic microbiology laboratories at Tygerberg Hospital (TBH) and Groote Schuur Hospital (GSH). Solid media used consisted of tryptose blood, boiled blood and MacConkey agar (for aerobic/ $\mathrm{CO}_{2}$-enriched conditions) and Brucella and/or tryptose blood agar (for anaerobic conditions). Liquid media used consisted of cooked meat medium or tryptic soy broth. Tissue samples were crushed, and crushed tissue and pus samples were inoculated onto the basic solid media listed prior to incubation. Tissue and pus samples were incubated on solid media only for at least 48 hours at TBH; a combination of solid media and broth was used at GSH, with solid media incubated for at least 48 hours and broth incubated for at least 120 hours, with subculture onto solid media if turbid. Pus swabs were incubated on solid media in $\mathrm{CO}_{2}$-enriched conditions for a minimum of 24 hours, with anaerobic incubation using solid media also included at GSH. Current local laboratory processing guidelines do not include the use of sonication or vortexing of the sample in the absence of submitted prosthetic material.

All pure cultures were identified. Mixed cultures were reviewed by a pathologist and followed up as appropriate. Identification and susceptibility testing were performed using the VITEK 2 automated system (bioMérieux, France) with supplemental rapid biochemical or antigen-based identification, and disc or gradient diffusion antibiotic susceptibility testing, as appropriate. Antibiotic susceptibility results were interpreted according to annually published Clinical Laboratory and Standards Institute guidelines. For the purposes of this article, organisms falling into the intermediate category were categorised as resistant, since antibiotic activity at the site of infection was likely to be suboptimal.

Statistical analysis was performed using Stata 16.1 (StataCorp, USA) and EpiCalc 2000 v1.02 (Brixton Books, UK). Parametric data are reported as means and standard deviations (SDs) with $95 \%$ confidence intervals (CIs) where appropriate. Non-parametric data are described as medians, interquartile ranges and ranges. Categorical data are described as frequencies and/or counts, with $95 \%$ CIs where appropriate.

\section{Results}

Between 1 March 2016 and 31 December 2019, 200 patients with chronic osteomyelitis of the appendicular skeleton underwent surgical treatment. No patients were excluded. The final cohort comprised 151 males and 49 females, with a mean (SD) age of 36.0 (14.3) years (range 10 - 84; 95\% CI 34.0 - 38.0) (Table 1). The distribution of affected anatomical sites is shown in Fig. 1.

\section{Microbial isolates}

Organisms were isolated from 169 patients (85\%), while no bacteria were isolated in 31 cases $(15 \%)$. A pure growth of a single organism was cultured from 130 patients (65\%), while polymicrobial infections were found in 39 cases (20\%), with two

\begin{tabular}{|c|c|}
\hline Variable & \\
\hline Age (years), mean (SD) & $36.0(14.3)$ \\
\hline Sex male, \% $(n)$ & $151(76)$ \\
\hline \multicolumn{2}{|l|}{ Original pathology, $\%(n)$} \\
\hline Fracture-related infection & $155(77)$ \\
\hline Haematogenous infection & $20(20)$ \\
\hline Contiguous infection & $5(3)$ \\
\hline \multicolumn{2}{|l|}{ Culture results, \% (n) } \\
\hline No growth & $31(16)$ \\
\hline Pure culture & $130(65)$ \\
\hline Polymicrobial (2 organisms) & $32(16)$ \\
\hline Polymicrobial (>2 organisms) & $7(4)$ \\
\hline \multicolumn{2}{|c|}{ Antibiogram available ( $N=218$ organisms), $\%(n)$} \\
\hline Yes & $215(99)$ \\
\hline No (not tested) & $3(1)$ \\
\hline
\end{tabular}

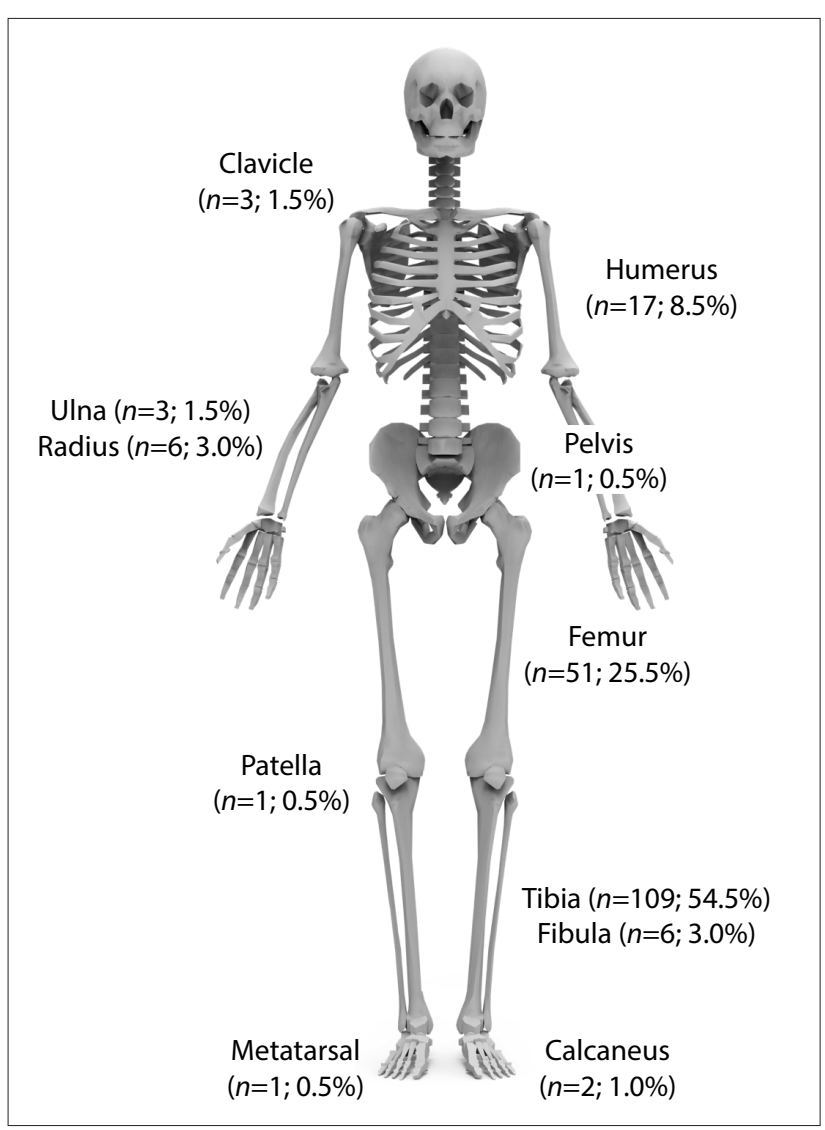

Fig. 1. Anatomical distribution of chronic osteomyelitis cases. 
distinct organisms isolated from 32 patients (16\%) and three or more organisms from 7 (4\%), giving a total of 218 isolates for analysis. Of these, 123 (56\%) were Gram-positive and 95 (44\%) were Gram-negative. A full breakdown of bacterial isolates is presented in Table 2.

The majority of the organisms $(n=174 / 218 ; 80 \%)$ were isolated from fracture-related infections. Gram-positives accounted for $53 \%(n=92 / 174)$ of the organisms from fracture-related infections. Members of the Enterobacterales contributed 55 isolates (32\%) in this context. The few cases of chronic osteomyelitis due to a contiguous source were caused by members of the Enterobacterales ( $n=4 / 5 ; 80 \%)$. For chronic osteomyelitis following haematogenous spread, Gram-positives also predominated $(n=30 / 39 ; 77 \%)$.

\section{Antibiograms}

Full antibiogram profiles were obtained for 215 bacterial isolates (99\%) (Tables 3 and 4). In 3 isolates (1\%), all of which were anaerobes, no antibiotic susceptibility testing was performed; these included 1 case of Clostridium tertium and 2 cases of Finegoldia magna.

Table 2. Organisms isolated from intraoperative samples in patients with chronic osteomyelitis, according to original pathology $(N=218)$

\begin{tabular}{|c|c|c|c|c|}
\hline & \multicolumn{3}{|c|}{ Original pathology } & \multirow[b]{2}{*}{ Total, $N$} \\
\hline & $\begin{array}{l}\text { Fracture-related } \\
\text { infections }(N=174), n\end{array}$ & $\begin{array}{l}\text { Haematogenous } \\
\text { osteomyelitis }(N=39), n\end{array}$ & $\begin{array}{l}\text { Contiguous } \\
\text { infection }(N=5), n\end{array}$ & \\
\hline \multicolumn{5}{|l|}{ Staphylococci } \\
\hline Methicillin susceptible Staphylococcus aureus & 37 & 24 & 1 & 62 \\
\hline Methicillin-resistant S. aureus & 20 & 2 & - & 22 \\
\hline Coagulase-negative staphylococci ${ }^{\star}$ & 6 & - & - & 6 \\
\hline \multicolumn{5}{|l|}{ Streptococci } \\
\hline Group A Streptococcus & 5 & 1 & - & 6 \\
\hline Group B Streptococcus & 3 & - & - & 3 \\
\hline Group C Streptococcus & 1 & - & - & 1 \\
\hline Group G Streptococcus & 1 & - & - & 1 \\
\hline S. constellatus & 1 & 1 & - & 2 \\
\hline \multicolumn{5}{|l|}{ Enterococci } \\
\hline Enterococcus faecalis & 9 & - & - & 9 \\
\hline Enterococcus species & 2 & - & - & 2 \\
\hline \multicolumn{5}{|l|}{ Aerobic Gram-positive bacilli } \\
\hline Corynebacterium striatum & 2 & 1 & - & 3 \\
\hline Corynebacterium species $^{\dagger}$ & 1 & 1 & - & 2 \\
\hline \multicolumn{5}{|l|}{ Enterobacterales } \\
\hline Proteus mirabilis & 17 & 3 & 2 & 22 \\
\hline P. hauseri & 1 & 1 & - & 2 \\
\hline P. penneri & - & 1 & - & 1 \\
\hline Enterobacter cloacae & 16 & - & - & 16 \\
\hline E. cancerogenus & 1 & - & - & 1 \\
\hline Klebsiella pneumoniae & 5 & 1 & - & 6 \\
\hline K. oxytoca & - & 1 & - & 1 \\
\hline K. aerogenes & - & - & 1 & 1 \\
\hline Serratia marcescens & 5 & - & - & 5 \\
\hline Providencia stuartii & 2 & - & - & 2 \\
\hline Escherichia coli & 1 & - & 1 & 2 \\
\hline Morganella morganii & 3 & - & - & 3 \\
\hline Citrobacter freundii/braakii & 2 & - & - & 2 \\
\hline Other ${ }^{\ddagger}$ & 2 & - & - & 2 \\
\hline \multicolumn{5}{|l|}{ Non-fermenting Gram-negative bacilli } \\
\hline Pseudomonas aeruginosa & 20 & - & - & 20 \\
\hline Acinetobacter baumannii & 6 & 1 & - & 7 \\
\hline Ralstonia pickettii & - & 1 & - & 1 \\
\hline \multicolumn{5}{|l|}{ Miscellaneous Gram-negative bacilli } \\
\hline Aeromonas hydrophlia/caviae & 1 & - & - & 1 \\
\hline \multicolumn{5}{|l|}{ Anaerobes } \\
\hline Clostridium tertium & 1 & - & - & 1 \\
\hline Finegoldia magna & 2 & - & - & 2 \\
\hline Bifidobacterium species & 1 & - & - & 1 \\
\hline Total & 174 & 39 & 5 & 218 \\
\hline
\end{tabular}


Table 3. Proportion of isolated organisms susceptible to tested antibiotics ${ }^{\star}$

\begin{tabular}{|c|c|c|c|c|}
\hline Antibiotic regimen & $\begin{array}{l}\text { Gram-positive isolates } \\
\text { tested, } n(N=123)\end{array}$ & $\begin{array}{l}\text { Susceptible Gram- } \\
\text { positive isolates, } n(\%)\end{array}$ & $\begin{array}{l}\text { Gram-negative isolates } \\
\text { tested, } n(N=95)\end{array}$ & $\begin{array}{l}\text { Susceptible Gram- } \\
\text { negative isolates, } n(\%)\end{array}$ \\
\hline Penicillin & 109 & $27(25)$ & - & - \\
\hline Ampicillin/amoxicillin & 104 & $28(27)$ & 37 & $10(27)$ \\
\hline Co-amoxiclav & - & - & 37 & $32(86)$ \\
\hline Cloxacillin $^{\dagger}$ & 114 & $81(71)$ & - & - \\
\hline Ciprofloxacin & 101 & $7(72)$ & 95 & $72(76)$ \\
\hline Co-trimoxazole & 91 & $66(73)$ & 75 & $43(57)$ \\
\hline Cefuroxime $^{*}$ & 103 & $81(79)$ & 67 & $28(42)$ \\
\hline Cefotaxime plus ceftriaxone ${ }^{\ddagger}$ & - & - & 67 & $58(87)$ \\
\hline Ceftazidime $e^{\ddagger}$ & - & - & 95 & $74(78)$ \\
\hline Cefepime & 104 & $82(79)$ & 95 & $75(79)$ \\
\hline Gentamicin & 116 & $82(71)$ & 95 & $78(82)$ \\
\hline Amikacin & - & - & 94 & $83(88)$ \\
\hline Erythromycin & 107 & $77(72)$ & - & - \\
\hline Clindamycin & 110 & $79(72)$ & - & - \\
\hline Vancomycin & 120 & $120(100)$ & - & - \\
\hline Piperacillin-tazobactam & - & - & 95 & $75(79)$ \\
\hline Ertapenem & - & - & 67 & $67(100)$ \\
\hline Imipenem & - & - & 77 & $59(77)$ \\
\hline Meropenem & - & - & 95 & $84(88)$ \\
\hline Colistin ${ }^{\S}$ & - & - & 23 & $23(100)$ \\
\hline Tigecycline & - & - & 35 & $33(94)$ \\
\hline Linezolid & 98 & $98(100)$ & - & - \\
\hline Rifampicin & 89 & $66(73)$ & - & - \\
\hline \multicolumn{5}{|c|}{ 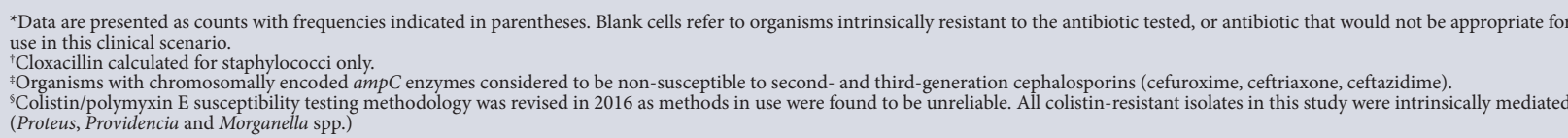 } \\
\hline
\end{tabular}

Key antibiotic susceptibility patterns for selected pathogens are summarised below.

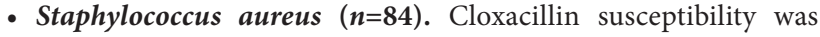
reported in 62 isolates (74\%), clindamycin susceptibility in 67 $(80 \%)$ and co-trimoxazole susceptibility in $60(71 \%)$. No reduced susceptibility to vancomycin was noted.

- Enterobacterales $(\boldsymbol{n}=\mathbf{6 6})$. Proteus species, specifically P. mirabilis, was the most frequently isolated species $(n=22 ; 33 \%)$, followed by Enterobacter cloacae $(n=16 ; 24 \%)$. Thirty isolates in the Enterobacterales group were identified as organisms typically harbouring chromosomally encoded class $\mathrm{C}$ beta-lactamases (45\%), and 9 were assumed to harbour extended-spectrum beta-lactamases according to Vitek 2 expert analysis (14\%). No Enterobacterale isolates with reduced susceptibility to the carbapenems were identified in this cohort.

- Pseudomonas aeruginosa ( $\boldsymbol{n}=\mathbf{2 0})$. The majority of $P$. aeruginosa isolates were susceptible to one or more first-line agents, with $80 \%$ of isolates testing susceptible to ceftazidime and cefepime, and $75 \%$ susceptible to piperacillin-tazobactam. All isolates were susceptible to amikacin, and $95 \%$ were susceptible to gentamicin. Ciprofloxacin resistance was observed in 6 isolates (30\%).

- Enterococci $(n=11)$. Nine species were ampicillin/amoxicillin susceptible (82\%). All isolates were susceptible to vancomycin.

- Beta-haemolytic streptococci $(\boldsymbol{n}=\mathbf{1 1})$. These organisms were assumed invariably to be susceptible to penicillin and vancomycin, or were susceptible when tested. Clindamycin resistance was observed in 2 isolates (18\%).
- Acinetobacter baumannii $(\boldsymbol{n}=7)$. Six of the 7 isolates (86\%) were resistant to all the antibiotics tested, except colistin, for which susceptibility was based on Vitek 2 . Tigecycline was only tested in 2 of these isolates, of which both were susceptible. One isolate tested susceptible to co-trimoxazole, gentamicin and tobramycin only.

The available resistance data were used to predict the potential efficacy of different empirical antibiotic monotherapy or combination regimens for bacterial isolates classified according to the original pathology (Table 5).

As more than one organism was cultured in 39 cases (20\%), the resistance data were used to predict potential efficacy of the different empirical antibiotic regimens on a per-patient basis (Table 6). In 6 patients (3\%), none of the proposed antibiotic combinations would be effective. Combinations of meropenem plus vancomycin and piperacillin-tazobactam plus amikacin plus vancomycin would both effectively treat $78 \%$ of patients with chronic osteomyelitis over all infective pathologies. Vancomycin and gentamicin provided predicted activity in $75 \%$ of patients, and a few locally available oral antibiotic combinations displayed similar predicted activity rates (co-amoxiclav plus ciprofloxacin 61\%, co-trimoxazole plus ciprofloxacin $61 \%$, ciprofloxacin plus rifampicin $61 \%$, ciprofloxacin plus cloxacillin $60 \%$ and ciprofloxacin plus clindamycin $60 \%$ ).

\section{Discussion}

The objective of this study was to review the antibiogram profiles of bacterial isolates from patients with chronic osteomyelitis of the 

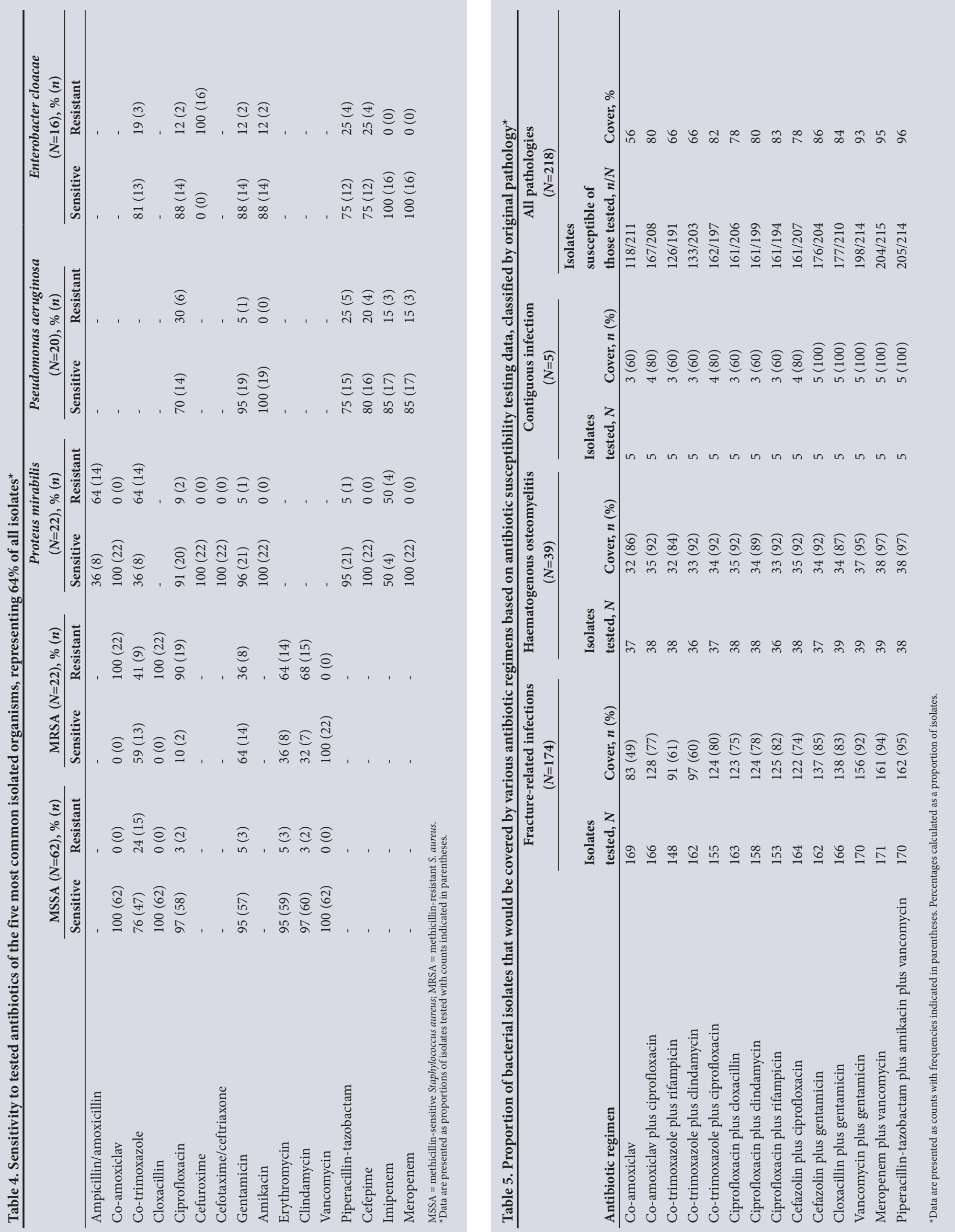
Table 6. Proportion of patients who would be covered by various antibiotic regimens based on antibiotic susceptibility testing data, classified by original pathology ${ }^{*}$

\begin{tabular}{|c|c|c|c|c|}
\hline Antibiotic regimen & $\begin{array}{l}\text { Fracture-related } \\
\text { infections }(N=155) \\
\text { cover, } n(\%)\end{array}$ & $\begin{array}{l}\text { Haematogenous } \\
\text { osteomyelitis } \\
(N=40) \text { cover, } n(\%)\end{array}$ & $\begin{array}{l}\text { Contiguous } \\
\text { infection }(N=5) \\
\text { cover, } n(\%)\end{array}$ & $\begin{array}{l}\text { Entire cohort } \\
(N=200), n(\%)\end{array}$ \\
\hline Co-amoxiclav & $55(35)$ & $27(68)$ & $2(40)$ & $84(42)$ \\
\hline Co-amoxiclav plus ciprofloxacin & $89(57)$ & $30(75)$ & $3(60)$ & $122(61)$ \\
\hline Co-trimoxazole plus rifampicin & $68(44)$ & $28(70)$ & $2(40)$ & $98(49)$ \\
\hline Co-trimoxazole plus clindamycin & $72(46)$ & $29(73)$ & $2(40)$ & $103(52)$ \\
\hline Co-trimoxazole plus ciprofloxacin & $89(57)$ & $29(73)$ & $3(60)$ & $121(61)$ \\
\hline Ciprofloxacin plus cloxacillin & $86(55)$ & $30(75)$ & $3(60)$ & $119(60)$ \\
\hline Ciprofloxacin plus clindamycin & $87(56)$ & $29(73)$ & $3(60)$ & $119(60)$ \\
\hline Ciprofloxacin plus rifampicin & $91(59)$ & $28(70)$ & $3(60)$ & $122(61)$ \\
\hline Cefazolin plus ciprofloxacin & $85(55)$ & $30(75)$ & $3(60)$ & $118(59)$ \\
\hline Cefazolin plus gentamicin & $99(64)$ & $29(73)$ & $4(80)$ & $132(66)$ \\
\hline Cloxacillin plus gentamicin & $101(65)$ & $29(73)$ & $4(80)$ & $134(67)$ \\
\hline Vancomycin plus gentamicin & $114(74)$ & $32(80)$ & $4(80)$ & $150(75)$ \\
\hline Meropenem plus vancomycin & $118(76)$ & $33(83)$ & $4(80)$ & $155(78)$ \\
\hline Piperacillin-tazobactam plus amikacin plus vancomycin & $119(77)$ & $33(83)$ & $4(80)$ & $156(78)$ \\
\hline
\end{tabular}

appendicular skeleton who underwent surgical treatment in two musculoskeletal infection units in the Western Cape, SA.

The main finding was that our results generally mirrored those of Sheehy et al. ${ }^{[8]}$ published in 2010 , which presented the bacteriological findings in 166 patients who underwent treatment at the Bone Infection Unit at the Nuffield Orthopaedic Centre in Oxford, UK, and which similarly described a wide range of bacterial isolates. In both studies, S. aureus was the most common bacterium isolated (32\% v. $39 \%$ in the present study), with similar proportions of methicillinresistant organisms identified ( $31 \%$ v. $26 \%$ in the present study). ${ }^{[8]}$ These results differ from a 2016 publication by Mthethwa and Marais ${ }^{[9]}$ reviewing the bacteriology of chronic osteomyelitis in KwaZulu-Natal Province, SA, which instead found the Enterobacterales to be the most common causative organisms (34\%).

Despite the lack of sonication processing strategies, which reportedly increase the bacteriological yield of intraoperative specimens, the current series (85\%) as well as the one by Mthethwa and Marais ${ }^{[9]}$ (93\%) showed higher organism culture yields than reported by Sheehy et al. ${ }^{[8]}(66 \%)$. Possible explanations include diagnostic criteria employed by the different centres, where Sheehy et al. ${ }^{[8]}$ only considered microbial isolates significant if they were returned from at least two separate samples. This strategy would exclude causative organisms where only one sample is sent, or could exclude obligate pathogens if only isolated from one sample. Conversely, this strategy prevents causation being ascribed to a potential contaminant/colonising organism isolated from a single sample. Some pathogens, such as $S$. aureus and S. pyogenes, are usually considered to be significant irrespective of the number of samples on which these isolates are identified. Another potential explanation for the increased yield of positive cultures is that, in our experience, patients with osteomyelitis in the developing-world setting are frequently directly referred to tertiary centres without initial treatment, which results in an antibiotic-free period at the time of presentation.

It is important to note that no antibiotic or antibiotic combination was consistently tested across all bacterial isolates, as susceptibility was inferred for some regimens (e.g. methicillin-sensitive S. aureus was assumed to be susceptible to co-amoxiclav and cephalosporins).
No proposed empirical antibiotic regimen would effectively treat all patients in this series. In terms of parenteral regimens, the combinations of meropenem plus vancomycin and piperacillintazobactam plus amikacin plus vancomycin (as a carbapenemsparing strategy) would both effectively treat $78 \%$ of cases in this cohort (mostly fracture-related chronic osteomyelitis). These combinations represent our current local protocol and are the two most effective parenteral empirical antibiotic regimens across all chronic osteomyelitis cases. The combination of meropenem plus vancomycin is the empirical antibiotic choice in the Oxford Bone Infection Unit. ${ }^{[8]}$ Predicted antibiotic activity was consistently higher for haematogenous chronic osteomyelitis cases than for fracturerelated infection.

The frequently used oral empirical antibiotic combination of co-trimoxazole plus rifampicin would only have been effective in $49 \%$ of cases, the empirical oral regimens of clindamycin plus ciprofloxacin in $60 \%$, co-trimoxazole plus ciprofloxacin in $61 \%$ and ciprofloxacin plus rifampicin in $61 \%$. Given the suboptimal predicted activity rates of these oral combinations, no empirical oral combination can be recommended for patients with chronic osteomyelitis at this time. Oral agents can and should still be used in accordance with antimicrobial stewardship guidelines and to facilitate outpatient management, but this should be targeted to individual patient culture. Parenteral regimens should be commenced and continued in the postoperative period until individual patient-level susceptibility testing data are available. Uncertainty remains in cases where wellcollected samples yield no significant growth, and these patients present considerable challenges to clinical teams. In these patients, the benefits and risks of broad-spectrum therapy must be weighed up against a watch-and-wait strategy, with repeated sampling as indicated.

Three anaerobic organisms were identified to species level. $C$. tertium is an anaerobic Gram-positive bacillus and is generally considered an uncommon pathogen in humans, although it has been isolated from post-traumatic musculoskeletal infections. ${ }^{[11,12]}$ F. magna is an anaerobic Gram-positive coccus that forms part of the normal flora of the skin and oral cavity. ${ }^{[13]}$ These organisms are mostly regarded as contaminants when isolated on culture, although 
they have been linked to a pathogenic role in prosthetic joint infection. ${ }^{[14,15]}$ Many laboratories do not perform susceptibility testing for anaerobic organisms, as the recommended methodology is highly specialised and/or not cost-effective. There is a concerning trend of increasing antibiotic resistance among anaerobic organisms globally, highlighting the need to validate simpler and less expensive methods for susceptibility testing and to conduct regular resistance surveys. ${ }^{[16]}$

Local antibiograms can also assist in developing empirical antibiotic regimens that can be continued in culture-negative chronic osteomyelitis. Failure to culture an organism could be explained by the presence of fastidious pathogens that have lost viability prior to culture, or by exposure to antibiotics, including those given preoperatively as surgical prophylaxis. In this series we failed to identify a pathogen in 31 cases (16\%) where chronic osteomyelitis was diagnosed through local signs (fistula or sinus, the presence of pus during surgery, and suggestive histological findings). These cases require an antibiotic regimen based on the most likely causative organisms. From this series it appears that oral combinations of ciprofloxacin plus cloxacillin (60\%), ciprofloxacin plus clindamycin (60\%), ciprofloxacin plus rifampicin (61\%) or ciprofloxacin plus co-trimoxazole $(61 \%)$ could be considered in culture-negative cases.

\section{Study limitations}

Several limitations limit the generalisability of this study, even within SA. These include the retrospective nature of the study design and the fact that the bacterial isolates originated from two institutions in the same city, which may not be representative of other geographical regions. Variability in specimen collection and laboratory processing techniques could have affected these findings, although these practices were fairly similar between the two institutions. Antibiotic susceptibility testing was not uniform between institutions, and inferences were made according to guidelines (e.g. beta-haemolytic streptococci at GSH were assumed to be susceptible to penicillin), hampering conclusive recommendations regarding optimal regimens across institutions. As only a single intraoperative sample was submitted in the majority of cases, organisms cultured were regarded as pathogens in all cases. Most patients in this cohort had fracture-related chronic osteomyelitis, limiting robust conclusions for the other two subgroups. Despite these limitations, this research represents the largest series of antibiograms of bacterial isolates from patients with chronic osteomyelitis in SA and can serve as a general guideline until similar findings from different regions are available. Further studies should investigate haematogenous and contiguous chronic osteomyelitis more thoroughly, should emphasise susceptibility testing for all oral agents available in our setting to draw appropriate conclusions, and should evaluate the impact of the empirical antibiotic choices on clinically relevant outcomes.

\section{Conclusions}

This study reports information regarding antibiogram profiles of chronic osteomyelitis bacterial isolates in the developing-world setting.
This information may assist decision-making regarding empirical antibiotic choices following definitive surgical management, and the combinations of meropenem plus vancomycin or piperacillintazobactam plus amikacin plus vancomycin (as a carbapenem-sparing strategy) are recommended. It is essential to repeat similar studies across settings to inform the development of national guidelines, and to repeat these studies periodically and revise guidelines accordingly.

\section{Declaration. None.}

Acknowledgements. The authors thank the patients and staff of the orthopaedic surgery departments at TBH and GSH, and the National Health Laboratory Service at both facilities, for facilitating this study.

Author contributions. NF: conception and design of research, acquisition of data, drafting and reading of manuscript; KR: acquisition of data, drafting and reading of manuscript; RGV: acquisition of data, drafting and reading of manuscript; $\mathrm{CMC}$ : acquisition of data, drafting and reading of manuscript; ML: conception and design of research, acquisition of data, drafting and reading of manuscript.

Funding. None.

Conflicts of interest. None.

1. Hall BB, Rosenblatt JE, Fitzgerald RH. Anaerobic septic arthritis and osteomyelitis. Orthop Clin North Am 1984;15(3):505-516. https://doi.org/10.1016/S0030-5898(20)31100-7

2. Haas DW, McAndrew MP. Bacterial osteomyelitis in adults: Evolving considerations in diagnosis and treatment. Am J Med 1996;101(5):550-561. https:///oi.org/10.1016/s0002-9343(96)00260-4

3. Marais LC, Ferreira N, Aldous C, le Roux TLB. The management of chronic osteomyelitis: Part I - Principles of post-infective reconstruction and antibiotic therapy. SA Orthop J 2014;13(3):32-39.

4. Walter G, Kemmerer M, Kappler C, Hoffmann R. Treatment algorithms for chronic osteomyelitis. Dtsch Arztebl Int 2012;109(14):257-264. https://doi.org/10.3238/arztebl.2012.0257

5. Simpson AH, Deakin M, Latham JM. Chronic osteomyelitis: The effect of the extent of surgical resection on infection-free survival. J Bone Joint Surg Br 2001;83(3):403-407. https://doi org/10.1302/0301-620x.83b3.10727

6. Cierny G. Surgical treatment of osteomyelitis. Plast Reconstr Surg 2011;127:190S-204S. https://doi. org $/ 10.1097 /$ prs.0b013e3182025070

7. Parsons B, Strauss E. Surgical management of chronic osteomyelitis. Am J Surg 2004;188(1A Parsons B, Strauss E. Surgical management of chronic os
Suppl):57-66. https://doi.org/10.1016/s0002-9610(03)00292-7

8. Sheehy SH, Atkins BA, Bejon P, et al. The microbiology of chronic osteomyelitis: Prevalence of . Sheehy $\mathrm{SH}$, Atkins BA, Bejon $\mathrm{P}$, et al. The microbiology of chronic osteomyelitis: Prevalence of
resistance to common empirical anti-microbial regimens. J Infect 2010;60(5):338-343. https://doi. resistance to common empirica
org/10.1016/j.jinf.2010.03.006

9. Mthethwa P, Marais L. The microbiology of chronic osteomyelitis in a developing world setting. SA Orthop J 2017;16(2):39-45. https://doi.org/10.17159/2309-8309/2017/v16n2a4

10. Metsemakers W, Morgenstern M, McNally MA, et al. Fracture-related infection: A consensus on definition from an international expert group. Injury 2018;49(3):505-510. https://doi.org/10.1016/j. injury.2017.08.040

1. Virot E, Servien E, Laurent F, et al. Reactivation of Clostridium tertium bone infection 30 years after the Iran-Iraq war. BMJ Case Rep 2015 (epub 18 February 2015). https://doi.org/10.1136/bcr-2014-209169

2. Gredlein CM, Silverman ML, Downey MS. Polymicrobial septic arthritis due to Clostridium species: Case report and review. Clin Infect Dis 2000;30(3):590-594. https://doi.org/10.1086/313686

13. Neumann A, Björck L, Frick I-M. Finegoldia magna, an anaerobic Gram-positive bacterium of the normal human microbiota, induces inflammation by activating neutrophils. Front Microbiol the normal human microbiota, induces inflammation
2020;11:65. https://doi.org/10.3389/fmicb.2020.00065

14. Levy P, Fenollar F, Stein A, Borrione F, Raoult D. Finegoldia magna: A forgotten pathogen in prosthetic Levy P, Fenollar F, Stein A, Borrione F, Raoult D. Finegoldia magna: A forgotten pathogen in prosthetic
joint infection rediscovered by molecular biology. Clin Infect Dis 2009;49(8):1244-1247. https://doi. joint infection rediscove
org $/ 10.1086 / 605672$

15. Söderquist B, Björklund S, Hellmark B, Jensen A, Brüggemann H. Finegoldia magna isolated from orthopedic joint implant-associated infections. J Clin Microbiol 2017;55(11):3283-3291. https://doi. org $/ 10.1128 / \mathrm{jcm} .00866-17$

16. Gajdács M, Spengler G, Urbán E. Identification and antimicrobial susceptibility testing of anaerobic bacteria: Rubik's cube of clinical microbiology? Antibiotics 2017;6(4):25. https://doi.org/10.3390/ antibiotics6040025

Accepted 23 February 2021 